\title{
Brazil nuts intake improves lipid profile, oxidative stress and microvascular function in obese adolescents: a randomized controlled trial
}

\author{
Priscila A Maranhão ${ }^{1}$, Luiz G Kraemer-Aguiar ${ }^{2 *}$, Cecilia L de Oliveira ${ }^{3}$, Maria CC Kuschnir ${ }^{4}$, Yasmine R Vieira ${ }^{1}$, \\ Maria GC Souza ${ }^{5}$, Josely C Koury ${ }^{6}$ and Eliete Bouskela ${ }^{7}$
}

\begin{abstract}
Background: Obesity is a chronic disease associated to an inflammatory process resulting in oxidative stress that leads to morpho-functional microvascular damage that could be improved by some dietary interventions. In this study, the intake of Brazil nuts (Bertholletia excelsa), composed of bioactive substances like selenium, $\alpha$ - e $\gamma$ tocopherol, folate and polyunsaturated fatty acids, have been investigated on antioxidant capacity, lipid and metabolic profiles and nutritive skin microcirculation in obese adolescents.

Methods: Obese female adolescents $(n=17), 15.4 \pm 2.0$ years and BMI of $35.6 \pm 3.3 \mathrm{~kg} / \mathrm{m}^{2}$, were randomized 1:1 in two groups with the diet supplemented either with Brazil nuts [BNG, $n=08,15-25 \mathrm{~g} /$ day (equivalent to 3 to 5 units/day)] or placebo [PG (lactose), $n=09$, one capsule/day] and followed for 16 weeks. Anthropometry, metabolic-lipid profiles, oxidative stress and morphological (capillary diameters) and functional [functional capillary density, red blood cell velocity (RBCV) at baseline and peak $\left(R B C V_{\max }\right)$ and time (TRBCV $\left.V_{\max }\right)$ to reach it during post-occlusive reactive hyperemia, after 1 min arterial occlusion] microvascular variables were assessed by nailfold videocapillaroscopy at baseline (T0) and after intervention (T1).
\end{abstract}

Results: T0 characteristics were similar between groups. At T1, BNG (intra-group variation) had increased selenium levels $(p=0.02), \operatorname{RBCV}(p=0.03)$ and $\operatorname{RBCV}_{\max }(p=0.03)$ and reduced total (TC) $(p=0.02)$ and LDL-cholesterol $(p=0.02)$. Compared to PG, Brazil nuts intake reduced TC $(p=0.003)$, triglycerides $(p=0.05)$ and LDL-ox $(p=0.02)$ and increased RBCV ( $p=0.03$ ).

Conclusion: Brazil nuts intake improved the lipid profile and microvascular function in obese adolescents, possibly due to its high level of unsaturated fatty acids and bioactive substances.

Trial Registration: Clinical Trials.gov NCT00937599

Keywords: microcirculation, obesity, Brazil nuts, adolescents, oxidative stress, lipid profile

\section{Background}

Worldwide prevalence of obesity in adolescence is actually high and increasing [1]. In Brazil, about $29 \%$ of adolescents tested between 2008-2009 have excessive weight [2]. Obesity, especially abdominal, even in young subjects leads to metabolic alterations, such as insulin

\footnotetext{
* Correspondence: gkraemer@ig.com.br

${ }^{2}$ Endocrinology, Department of Internal Medicine; Clinical and Experimental Research Laboratory in Vascular Biology - BioVasc; Rua São Francisco Xavier 524, Rio de Janeiro, CEP:20550-013 - Brazil

Full list of author information is available at the end of the article
}

resistance and dyslipidaemia, increasing risk factors for cardiovascular disease (CVD) in adulthood [3].

Excessive abdominal adiposity is characterized by accumulation of adipose tissue and is associated to a lowgrade inflammatory process and oxidative stress, both well-established pathogenetic factors for cardiovascular diseases [4]. Morpho-functional microvascular alterations related to metabolic disorders have already been described and findings on skin were related to microvascular dysfunction on coronary bed [5], occurring even in the absence of disglycaemic states [6] pointing to obesity per se as independent risk factor for microangiopathy.

\section{C) Biomed Central}


Possibly, longer duration of excessive adiposity is involved on it as well. Recently, we have unraveled that overweight/obese young women have microvascular dysfunction linked to adiposity levels and glucose homeostasis [7]. Certainly, obesity in adolescence and its long-term damage to target organs in adulthood deserves special focus and strategies to reduce future cardiovascular risks.

Excessive visceral fat induces the release of cytokines, such as tumor necrosis factor-alfa (TNF $\alpha$ ) and interleukin-6 (IL-6) leading to increased production of reactive oxygen species (ROS) and subsequent induction of tissue oxidative stress [8]. Some authors consider this pathophysiological process as a major mechanism underlying cardiovascular obesity-related comorbidities [9]. The antioxidant system is directly linked to environmental factors and nutrient intake. Some minerals such as selenium are also involved in decreasing levels of hydrogen peroxide and reducing lesions to cellular membranes [10]. Altered metabolic pathway of very low density lipoprotein cholesterol (VLDL) secondary to excessive visceral adiposity results on higher levels of low density lipoprotein (LDL) particles, which are more aggressive to endothelium. Additionally, the oxidative stress increases oxidation of these LDL particles [11], a process identified as a risk factor for atherosclerosis [12].

Bioactive substances existing in nuts have already been identified [13] and their beneficial effects on inflammation [14] and on endothelial function [15] demonstrated. The Brazil nut (Bertholletia excelsa) comes from the Amazon region and has a complex matrix, composed of bioactive substances, such as selenium, $\alpha$ - e $\gamma$ - tocopherol, phenolic compounds, folate, magnesium, potassium, calcium, proteins and mono (MUFA) and polyunsaturated (PUFA) fatty acids [14]. Its composition is different from other nuts and data to corroborate its beneficial effects, especially in obese adolescents with special focus on microcirculatory function are lacking.

The aims of this study were to investigate the influence of Brazil nuts consumption on nutritive skin microcirculation, serum antioxidant capacity, lipid and metabolic/cardiovascular risk profiles in obese female adolescents.

\section{Subjects and Methods Study Population}

Seventeen female adolescents $(15.4 \pm 2.0$ years $)$ were selected, after spontaneous interest to participate (male adolescents showed very little interest), from outpatient care clinics for Prevention and Assistance on Cardiovascular and Metabolic Disease in Adolescence (NESA, State University of Rio de Janeiro, Brazil). Main inclusion criterion was being above $95^{\text {th }}$ percentile for BMI according to age [16]. Main exclusion criteria were: use of any nutritional intervention and/or drugs; presence of chronic diseases (diabetes mellitus and/or hypertension) or lactose intolerance; a verbally informed weight reduction six months before entering the study; being beyond stage IV for Tanner pubertal development [17] and dietary habits of an excessive consumption of any kind of nut. All volunteers gave their written informed consent and this study was approved by the Ethics Committee for Clinical Research of Pedro Ernesto University Hospital (COEP 1950/2007).

\section{Experimental Design}

The study was a 16 -week non-blinded pilot trial with two randomly selected groups of obese female adolescents: Brazil nut (BNG, $\mathrm{n}=08$ ) ingested 15-25 g/day (equivalent to 3 to 5 units/day) of Brazil nuts and placebo ( $\mathrm{PG}, \mathrm{n}=09$ ) one capsule/day containing lactose. Lactose was chosen as placebo due to its lack of therapeutic effects to improve adolescents' compliance to the study. Both groups were informed that they would receive a supplemental dietary intake composed of Brazil nuts but only one group would receive it on its natural form. Nuts were consumed as snacks or with meals in salads. Before the beginning and at the end of the study, the usual food intake of each participant was assessed by a dietary inquiry and, during the study, adolescents were advised not to change their dietary habits.

Anthropometry, blood, urine and microvascular parameters were analyzed at baseline (T0) and after 16 weeks (T1).

\section{Brazil Nuts Diet}

Brazil nuts (Bertholletia excelsa) consumption was calculated to achieve $10 \%$ of the energy from MUFAs in the diet. Total energy intake was calculated by energy expenditure to overweight children and adolescents of 3 to 18 years according to dietary reference intake (DRI). Adolescents received 15-25 g/day (equivalent to 3 to 5 units/ day) in bags. Serum selenium levels and returned empty bags were used as markers of compliance for BNG.

Brazil nut composition was determined in $100 \mathrm{~g}$ by Adolph Lutz Institute-Brazil assays (1985). Lipid, carbohydrates and protein content (mean \pm SD) was $50.6 \pm$ $0.08 \mathrm{~g}$ [coefficient of variation (CV) 1.6\%], $25.9 \pm 0.6 \mathrm{~g}$ (CV 2.4\%) and $16.8 \pm 0.2 \mathrm{~g}$ (CV 1.4\%). Selenium was measured by flame atomic absorption spectrometry. Saturated (SFA - 15.3 g), monounsaturated (MUFA $27.4 \mathrm{~g}$ ) and polyunsaturated (PUFA) fatty acids (21 g) contents were calculated according to Brazilian Table of Food Composition [18]. Therefore, nut intake (15-25 g/day) was about: $124 \pm 31 \mathrm{kcal}$ with $5.2 \pm 1.3 \mathrm{~g}$ of carbohydrates and $10.1 \pm 2.5 \mathrm{~g}$ of lipids. The latter was composed of $4.2 \pm 1.0 \mathrm{~g}$ of PUFA, $5.5 \pm 1.4 \mathrm{~g}$ of MUFA and $3.0 \pm 0.7 \mathrm{~g}$ of SFA and $108.5 \pm 27 \mu \mathrm{g}$ of selenium. 


\section{Anthropometry}

The same trained examiner collected anthropometric measurements in duplicate, waist circumference, height, weight as previously reported [19]. BMI was defined as the ratio between weight in $\mathrm{kg}$ and squared height in meters.

\section{Laboratory Analysis}

All laboratory measurements were performed in duplicate after 10-12 hours fast using an automated method (Modular Analytics E 170 and P, Roche, Basel, Switzerland). Fasting plasma glucose (FPG), total cholesterol (TC), triglycerides (TG) and high-density lipoprotein (HDL) cholesterol were measured respectively, by enzyme-colorimetric GOD-PAP [inter-assay coefficient of variation $(\mathrm{IACV})=1.09 \%]$, enzymatic GPO-PAP $(\mathrm{IACV}=2.93 \%)$, enzymatic GPO-PAP $(\mathrm{IACV}=1.29 \%)$ and enzyme-colorimetric without pre-treatment (IACV $=3.23 \%)$. Plasma LDL-cholesterol was calculated according to Friedwald equation [20]. C-reactive protein and serum insulin were respectively measured by imunoturbidimetry (IACV $=8 \%$ ) and eletrochemiluminescence $(\mathrm{IACV}=10.6 \%)$. Homeostasis model assessment (HOMAIR) was calculated (fasting serum insulin $(\mu \mathrm{UI} / \mathrm{ml})$ X FPG $(\mathrm{mmol} / \mathrm{l}) / 22.5)$. Serum antioxidant capacity was determined by glutathione peroxidase (GPx) through Elisa [GPX3 (human) Elisa Kit, Axxora, LLC, USA, diluted in $1: 200$ (IACV $=3.9 \%$; sensitivity $=0.1 \mathrm{ng} / \mathrm{ml}]$. OxidizedLDL (ox-LDL) levels were also analyzed through Elisa (Kit Mercodia, Sweden), diluted in 1:6561 (IACV = 6.13\%; sensitivity $=0.05 \mathrm{ng} / \mathrm{ml}$ ). Oxidative stress was measured by a competitive enzyme-linked immunosorbant assay in duplicate determining levels of isoprostane nominated as 8-epi-prostaglandin $\mathrm{F}_{2 \alpha}$ (8-epi-PGF $\mathrm{F}_{2 \alpha}$ ) in urine samples (BIOXYTECH urinary 8-epi-PGF $2 \alpha$ - OxisResearch, Portland, OR, USA). Urinary samples were acidified during collection with $\mathrm{HCl} 6 \mathrm{~mol} / \mathrm{l}$ (final $\mathrm{pH}=2.0$ ), diluted to $1: 2$ before measurements and results were corrected for creatinine levels in each sample assessed by enzymatic colorimetric Modular Evo (Roche) with an intra-assay CV of 5\% (2-14\%) [19] Serum selenium was determined through atomic absorption spectrometry using a SpectraAA - 640Z - VARIAN (IACV = 11\%).

\section{Microvascular Function Assessment}

Nailfold videocapillaroscopy (NVC) was carried out and analyzed according to a standardized, well-validated methodology on the $4^{\text {th }}$ finger of the left hand [7]. The exam, always made by the same observer who was not aware of any patient data, recorded continuously microvascular parameters for later measurements using the Cap Image software [21]. Functional capillary density (FCD), number of capillaries $/ \mathrm{mm}^{2}$ with flowing red blood cells, was evaluated using x250 magnification and an area of $3 \mathrm{~mm}$ of the distal row of capillaries into three different areas [Intra-assay coefficient of variation - IACV $=5.5 \pm 2.5 \%$ ]. Capillary diameters [afferent (AF), apical (AP) and efferent (EF)], red blood cell velocity $(\mathrm{RBCV})$ at rest, after 1 min arterial occlusion $\left(R B C V_{\max }\right)$ and time taken to reach it $\left(\mathrm{TRBCV}_{\max }\right)$ were measured, with a final magnification of $\mathrm{x} 680$, before and during the post-occlusive reactive hyperemia (PORH) response after $1 \mathrm{~min}$ ischemia. Before RBCV assessment on an individual capillary loop, a pressure cuff $(1 \mathrm{~cm}$ wide) was placed around the proximal phalanx and connected to a mercury manometer. Conceptually, AF, AP and EF are considered morphological and FCD, RBCV, $\mathrm{RBCV}_{\text {max }}$ and $T R B C V_{\text {max }}$ functional microcirculatory parameters. Capillary diameters and basal RBCV were measured three times each and IACV for all measurements ranged from 16.9 to $17.1 \%$. At PORH, each variable was tested once. NVC was repeated on nine subjects in different days and the IACV ranged from $12.3 \%$ to $17.3 \%$ and from $2.0 \%$ to $9.0 \%$ between morphological and functional parameters, respectively.

\section{Statisticals Analysis}

Data are expressed as median $\left[1^{\text {st }}-3^{\text {rd }}\right]$ and analyzed by Graphpad Prism 4.0, 2003 and intra- or inter-group analysis compares significant results in different time points within the same group or between BNG and PG, respectively. Comparisons between groups at T0 and T1 and intra-group differences were determined using Mann-Whitney U test and Wilcoxon matched pair tests, respectively. GPower 3.1.10 software was used for power analysis and sample size estimation. The statistical power for comparisons between two dependent groups was above 0.9 with an a error probability of 0.01 for RBCV, estimating a total sample size of 7 patients/group. Significant differences were assumed to be present at $p<0.05$.

\section{Results}

Adolescents included in the study had $15.4 \pm 2.0$ years and BMI of $35.6 \pm 3.3 \mathrm{~kg} / \mathrm{m}^{2}$. At T0, there were no significant differences between groups (inter-group) on anthropometrical-laboratorial-microvascular variables (tables 1, 2 and 3). On the counterpart, at T1, we observed lower values for total $(p=0.003)$ and LDLcholesterol $(p=0.03)$, and also for TG $(p=0.05)$ for BNG compared to PG.

Body mass, BMI, waist circumference and metabolic profile were all kept unchanged during the follow-up on both groups. Additionally, we have noticed that Brazil nuts consumption did not influence glucose homeostasis (FPG, insulin, HOMA-IR) or CRP levels, although its positive influence was observed on LDL- $(p=0.01)$ and total cholesterol $(p=0.01)$ levels (table 1$)$. 
Table 1 Anthropometric measurements and metabolic profile of obese female adolescents at baseline and after 16 weeks of Brazil nuts (BNG) or placebo (PG) intake

\begin{tabular}{|c|c|c|c|c|}
\hline & \multicolumn{2}{|c|}{ BNG } & \multicolumn{2}{|c|}{ PG } \\
\hline & T0 & T1 & TO & $\mathrm{T} 1$ \\
\hline Body mass $(\mathrm{kg})$ & 86.3 [82.2-94.3] & $86.3[80.3-96.9]$ & 91.7 [82.1-110.8] & 93.2[83.7-108.7] \\
\hline Height (m) & $1.55[1.52-1.66]$ & $1.55[1.53-1.67]$ & $1.64[1.56-1.69]$ & $1.64[1.56-1.69]$ \\
\hline BMI $\left(\mathrm{kg} / \mathrm{m}^{2}\right)$ & $35.3[33.9-36.0]$ & $35.3[33.3-36.2]$ & $34.0[33.0-39.1]$ & $35.6[32.8-38.9]$ \\
\hline Waist circumference $(\mathrm{cm})$ & 105.0[93.0-117.8] & 112.0[99.5-116.0] & 111.0[104.0-117.8] & $115.0[107.5-120.0]$ \\
\hline Insulin (mcU/ml) & $15.9[14.5-23.6]$ & $15.5[12.2-22.2]$ & 18.0[15.2-20.1] & 18.0[10.9-22.8] \\
\hline Fasting glucose (mg/dl) & $89.5[81.5-94.0]$ & $86.0[82.5-93.0]$ & 87.0[82.0-90.5] & $90.0[82.0-94.0]$ \\
\hline HOMA & $3.6[2.9-5.2]$ & $3.5[2.6-6.5]$ & $3.9[3.1-4.4]$ & $3.7[2.2-5.2]$ \\
\hline CRP (mg/dl) & $0.31[0.15-0.63]$ & $0.37[0.20-0.68]$ & $0.38[0.14-0.78]$ & $0.52[0.29-0.79]$ \\
\hline Cholesterol (mg/dl) & $152.0[140.5-159.0]$ & $136.0[129.5-141.5] *+$ & $167.0[133.5-184.5]$ & $170.0[148.5-177.0]$ \\
\hline HDL-c (mg/dl) & $44.0[41.5-48.5]$ & $43.5[40.0-50.5]$ & $45.0[36.0-48.0]$ & $45.0[41.0-47.5]$ \\
\hline LDL-c (mg/dl) & $86.5[80.5-98.0]$ & 72.5[71.0-83.0] ${ }^{*}+$ & 106.0[77.0-123.0] & 103.0[87.0-109.0] \\
\hline TG (mg/dl) & $84.5[68.0-98.5]$ & $69.0[58.5-83.5] \dagger$ & $113.5[87.0-132.5]$ & 106.0[77.0-153.0] \\
\hline
\end{tabular}

* significant results intra-group $(p<0.05)$

† significant results inter-group $(p<0.05)$

At T0, antioxidant capacity biomarkers were similar between groups. Gpx, LDL-ox and 8-epi-PGF $\mathrm{PF}_{2 \alpha}$ did not change during the follow-up in either group (Table 2). It should be highlighted that at $\mathrm{T} 1$, serum levels of LDL-ox were lower on BNG when compared to PG ( $p=$ 0.02), while on PG, serum selenium levels, a marker of compliance for the proposed intervention, was kept unchanged [118 (107.5-148.5 $\mu \mathrm{g} / \mathrm{l})$ vs. 126 (106.5-146.5 $\mu \mathrm{g} / \mathrm{l}), p=0.74]$ but on BNG we have observed a significant increase [110.5 (87.5-131.5 $\mu \mathrm{g} / \mathrm{l})$ vs. 133 (104.5-178 $\mu \mathrm{g} / \mathrm{l}), p=0.02$ ] (Figure 1).

Morphological microvascular parameters (capillary diameters - Table 3) did not change during the follow-up in both groups. Obese female adolescent in PG had all functional microvascular parameters unaltered during the follow-up but those supplemented with Brazil nuts (BNG) increased RBCV and $\mathrm{RBCV}_{\max }$ during PORH $13.9 \%$ and $9.52 \%$, respectively (intragroup results) (Figure 2). Additionally, RBCV at T1 was higher on BNG when compared to PG at the same time point [1.63(1.6-1.7) vs. $1.54(1.4-1.56) \mathrm{mm} / \mathrm{s}$; $p=0.02]$.

\section{Discussion}

Obesity is associated to metabolic disturbances, including insulin resistance, dyslipidemia and low-grade inflammation, all of them putative factors for endothelial and microvascular dysfunction (MD). Frequent nut intake is associated with many health benefits in adults [22-24], although no studies on adolescents could be found. To the best of our knowledge, this study is the first one to investigate effects of Brazil nuts intake on metabolic-lipid profiles, antioxidant status and microvascular parameters in obese female of this specific age period.

Nuts are rich in lipids, mainly in MUFAs and PUFAs and have high energy density. Comparing fat composition of diets rich on SFAs, high content of MUFAs and PUFAs in foodstuffs is potentially beneficial to health [25]. In spite of the caloric composition, some authors have demonstrated that consumption of nuts for short periods (less than 4 weeks) did not increase body mass [26]. In the present study, both groups kept body mass, waist circumference and BMI unchanged suggesting that although Brazil nuts have high energy density, possibly

Table 2 Biomarkers with antioxidant capacity in obese female adolescents at baseline and after 16 weeks of Brazil nuts (BNG) or placebo (PG) intake

\begin{tabular}{|c|c|c|c|c|}
\hline & \multicolumn{2}{|c|}{ BNG } & \multicolumn{2}{|c|}{ PG } \\
\hline & T0 & T1 & T0 & T1 \\
\hline 8-epi-PGF $2 \alpha(\mathrm{pg} / \mu \mathrm{mol} / \mathrm{g}$ of creatinine $)$ & $143.2[102.9-198.8]$ & $100.0[80.0-131.4]$ & $34.0[61.5-126.6]$ & $88.4[65.0-149.5]$ \\
\hline LDL-ox (ng/ml) & $622.4[457.2-665.0]$ & 514.9[440.3-624.6]† & 648.8[515.9-737.9] & $646.9[595.7-883.5]$ \\
\hline GPX-3 (ng/ml) & $15.5[12.4-19.8]$ & 16.7[12.8-17.3] & $17.2[13.7-19.7]$ & $17.3[13.9-20.1]$ \\
\hline Selenium ( $\mu \mathrm{g} / \mathrm{L})$ & $110.5[87.5-131.5]$ & 133.0[104.5-178.0] * & $118.0[107.5-148.5]$ & $126.0[106.5-146.5]$ \\
\hline
\end{tabular}

* Signicant intra-group results $(p<0.05)$.

† Signficant inter-group results $(p<0.05)$. 
Table 3 Microcirculatory parameters on obese female adolescents at baseline and after 16 weeks of Brazil nuts (BNG) or placebo (PG) intake

\begin{tabular}{lllll}
\hline & \multicolumn{1}{c}{ BNG } & \multicolumn{1}{c}{ PG } \\
\hline Functional capillary density $\left(\mathbf{n} / \mathbf{m m}^{2}\right)$ & T0 & T1 & T0 & T1 \\
Afferent diameter $(\boldsymbol{\mu m})$ & $10.2[7.8-11.2]$ & $8.8[8.2-11.8]$ & $9.9[8.0-17.4]$ & $10.2[8.4-13.4]$ \\
Apical diameter $(\boldsymbol{\mu m})$ & $15.7[13.9-18.6]$ & $15.2[11.8-19.5]$ & $15.1[12.9-18.6]$ & $14.0[11.6-16.1]$ \\
Efferent diameter $(\boldsymbol{\mu m})$ & $19.7[18.1-22.3]$ & $22.1[18.2-24.1]$ & $21.9[18.5-27.5]$ & $20.0[18.4-25.6]$ \\
RBCV $(\mathbf{m m} / \mathbf{s})$ & $20.4[17.5-22.9]$ & $21.6[19.2-25.3]$ & $18.4[15.9-21.5]$ & $21.5[17.8-21.8]$ \\
RBCV $_{\max }(\mathbf{m m} / \mathbf{s})$ & $1.43[1.38-1.48]$ & $1.63[1.6-1.7]{ }^{*}+$ & $1.47[1.41-1.52]$ & $1.54[1.40-1.56]$ \\
TRBCV $_{\max }(\mathbf{s})$ & $1.68[1.64-1.73]$ & $1.84[1.81-1.94]$ & $1.71[1.56-1.79]$ & $1.79[1.76-1.84]$ \\
\hline
\end{tabular}

* Significant results intra-group $(p<0.05)$.

† Significant results inter-group $(P<0.05)$.

the satiety feeling due to nuts composition, mediumchain-triglycerides [27], fiber and protein [26] reduced energy intake from other sources. Positive influences on lipid profile by other types of nuts have been already demonstrated [28]. It has been reported that consumption of Brazil nuts during 15 days did not improve LDLand total cholesterol and its benefits were noticed on transfer of cholesterol into HDL pool. In our study, supplementation of Brazil nuts during 16 weeks to obese female adolescents positively influenced the lipid profile such as, total cholesterol (TC), LDL-C and TG, but the present study did not assess cholesteryl esters.

MD has already been described in obesity [29], metabolic syndrome at normoglycemia [19] and type 2 diabetes mellitus using NVC. This technique is a noninvasive method to assess microvascular morphology and function and data obtained using this diagnostic tool has been already associated to cardiovascular risk [30]. In our investigation, adolescents were seen every 4 weeks what is roughly during the same phase of the menstrual cycle and no especial precaution were taken in this direction because it has already been shown that microvascular function is not dependent on menstrual cycle in ovulatory women [31]. Even tested for shortterm period in obese female adolescents, we could observe an improvement of microvascular reactivity by supplementing their diet with Brazil nuts and to our knowledge this is the first clinical study that showed its

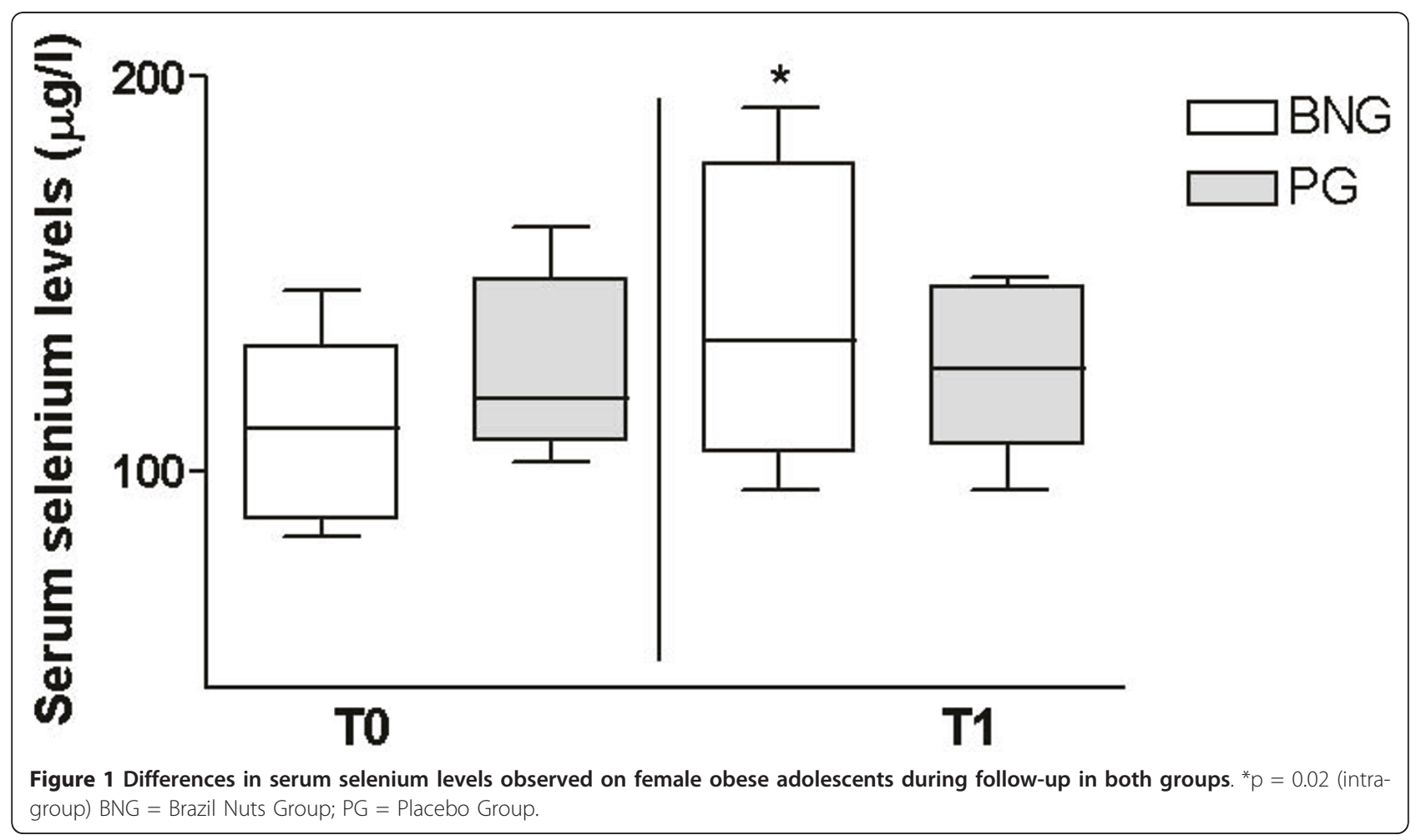



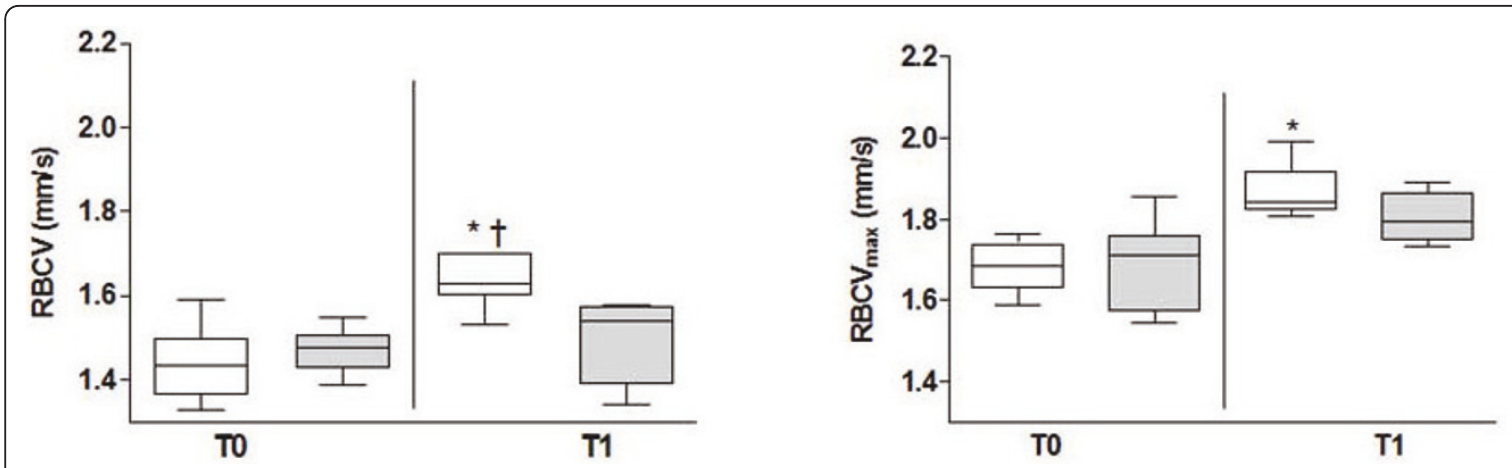

Figure 2 Resting Red Blood Cell Velocity (RBCV) and $\mathrm{RBCV}_{\max }$ during post-occlusive reactive hyperemia response (PORH) on obese female adolescents at baseline and after 16 weeks of Brazil nuts (BNG) or placebo (PG) intake. ${ }^{*} p=0.03$ (intra-group) and $+p=0.03$ (inter-group).

positive influence on nutritive skin microvascular function, although the effects of PUFAs and MUFAs contained in nuts have been well established on macro and microvascular functions [15].

Capillary morphology was not influenced by Brazil nuts consumption. The analysis of the hemodynamic behavior of the microcirculation was pursued by measuring RBCV before and after 1 min arterial occlusion as well as the time taken to reach $\mathrm{RBCV}_{\max }$. We have chosen $1 \mathrm{~min}$ arterial occlusion because our purpose was to measure the effect of an increased shear stress during the reactive hyperemia response and to decrease the discomfort for our patients $(1 \mathrm{~min}$ ischemia is more tolerable than 4-6 $\mathrm{min}$ ) [32]. Longer duration of the occlusion is commonly used to evaluate capillary recruitment [33]. In the BNG, a significant improvement on $\mathrm{RBCV}$ at baseline and during PORH could be detected in conjunction with a trend towards a positive influence on time for reperfusion $\left(\mathrm{TRBCV}_{\max }\right)$, which needs further elucidation. PORH is thought to be determined at the level of small arterioles [34] and to be independent of the autonomic nervous system [35]. After the onset of reperfusion there is a sharp rise in blood flow followed by a gradual return to its baseline level, influenced by accumulation of vasodilator metabolites (including nitric oxide) and formation of ROS, normally washed out or destroyed by circulating blood and smooth muscle cell reactivity. During reperfusion, the myogenic response, due to rapid stretch of microvascular smooth muscle cells, is responsible, at least partially, for the return of blood flow to its baseline values. Our sample size did not allow us to correlate variables to find out associations between them, but we suppose that higher intake of MUFAs and PUFAs by obese adolescents on BNG resulting in an improved lipid profile and lower levels of LDL-ox, positively influenced microvascular reactivity. To strengthen this hypothesis we have previously noticed a multiple association between total and LDL-cholesterol levels and capillary microflow on obese metabolic syndrome subjects [19], suggesting that interventions with beneficial effects on the lipid profile could positively influence RBCV and $\mathrm{RBCV}_{\max }$. Consumption of nutrients rich in PUFAs (especially linolenic acids) such as fish oil and also Brazil nuts was associated to reduction in fibrinogen levels and in blood viscosity [36].

Nuts as sources of MUFAs have also been inserted in diets aiming to improve not only lipid profile but also insulin sensitivity, but in healthy, type 2 diabetes mellitus patients [37], and our own results in obese adolescents, nuts-enriched diets did not influence glucose homeostasis and CRP, an acute-phase protein, associated to atherosclerosis, metabolic disorders and CV disease [38].

Brazil nuts consumption during 15 days increased selenium levels, showing that shorter periods with higher amounts of nuts per day were followed by increments of $370 \%$ on selenium levels [24]. Physiological values for selenium ranged from 53 to $161.1 \mu \mathrm{g} / \mathrm{l}$ [39]. In the present study, selenium levels increased on BNG and were considered as a marker of good compliance although a significant difference could not be found inter-group (perhaps the intervention time was too short). Oxidative imbalance has been pointed as a causal factor for CVD. Nutrients and bioactive substances with antioxidant action are able to avoid arachidonic acid oxidation [33] and improvement of lipid peroxidation could reduce plasmatic and urinary levels of 8 -epi-PGF $\mathrm{PG}_{2 \alpha}$, an oxidative product of arachidonic acid related to cell membranes. Urinary 8-epi-PGF $2 \alpha$ levels, also called isoprostanes, have shown large variation already at baseline inter- (two adolescents at PG had reduced urinary 8-epi$\mathrm{PGF}_{2 \alpha}$ levels) and also intra-group in a reduced sample size and no significant changes could be detected on either group. However, we have noticed that its levels in BNG showed a decremental direction while in PG results went in the opposite way. It should be recalled that isoprostanes, derived from the arachidonic acid, are only 
one form of metabolite related to oxidative stress thus not able to reflect the whole oxidative status on cell membranes. Although our data do not explain improvements in the nutritive microvascular reactivity by an amelioration of urinary isoprostanes, we suggest that this pathophysiological focus deserves further elucidation with the use of other biomarkers of oxidative stress.

While investigated markers of oxidative stress were not altered by Brazil nuts consumption (Table 2), we could notice that LDL-ox levels were significantly reduced in BNG, probably due to reduction on oxidative stress status. The exact mechanism involved on it was not our aim, but the knowledge that LDL-ox is associated to endothelial dysfunction and atherosclerosis rise possible questions about the beneficial long-term effects of Brazil nuts consumption on obesity.

Limitations of the present study warrant mention. Although a power analysis was done to strengthen our results, the reduced number of patients investigated limits our conclusion to the population studied. Although the present study was randomized, it was not blinded and we have to consider that some changes in diet that we were not aware of, might have happened. Long-term beneficial cardiovascular outcomes could be inferred by these data.

Finally, treatment strategies for obese adolescent should focus on lifestyle interventions, aiming weight reduction, even if some supplements with established beneficial effects are added to diets.

\section{Conclusion}

Our results show that short-term intake of Brazil nuts added to diet of an obese female adolescent group did not change body mass or waist circumference, but, as a nutrient rich in bioactive substances, it positively influenced lipid profile and nutritive microvascular reactivity.

\footnotetext{
List of Abbreviations

8-epi-PGF $2 a$ : (8-epi-prostaglandin $F_{2 a}$ ); AF: (Afferent diameter); AP: (Apical diameter); BMI: (Body mass index); BNG: (Brazil Nut group); BP: (Blood pressure); CRP: (C-reactive protein); CV: (Coefficient of variation); CVD: (Cardiovascular disease); EF: (Efferent diameter); FCD: (Functional capillary density); FPG: (Fasting plasma glucose); Gpx: (Glutathione peroxidase); HDL: (High-density lipoprotein); HOMA-IR: (Homeostasis model assessment for insulin resistance); IACV: (Inter-assay coefficient of variation; IL-6: (interleukin6); IR: (Insulin resistance); LDL: (Low-density lipoprotein); MUFA:

(Monousatured fatty acids); NCHS: (National Center of healthy Statistics); NVC: (Nailfold videocapillaroscopy); PG: (Placebo group); PORH: (Post-occlusive reactive hyperemia); PUFA: (Polyunsatured fatty acids); RBCV: (Red blood cell velocity); $\mathrm{RBCV}_{\text {max }}$ (Peak red blood cell velocity); ROS: (Reactive oxygen species); SFA: (Saturated fatty acids); TC: (Total Cholesterol); TG: (Triglycerides); TNFa: (Tumor necrosis factor-alfa); TRBCV $V_{\text {max }}$ (Time taken to reach $\left.\mathrm{RBCV}_{\text {max }}\right)$; VLDL: (Very low density lipoprotein); $\omega$-3: (Linolenic fatty acids); $\omega-6$ : (Linoleic fatty acids).
}

\section{Acknowledgements}

Authors would like to thank Drs. Tatiane Bertoni de Toledo and Fabiana Barreto Lima for their help in recruiting patients and EMBRAPA for selenium measurements and Funding: National Research Council (CNPq), Foundation to Support Research in the State of Rio de Janeiro (FAPERJ) and Agency to Finance Studies and Projects (FINEP). During this study Ms. Priscila Maranhão received a fellowship from the Coordination to Improve Graduate Personnel (CAPES).

\section{Author details}

${ }^{1}$ Clinical and Experimental Research Laboratory in Vascular Biology - BioVasc; Rua São Francisco Xavier, 524, Rio de Janeiro, CEP:20550-013 - Brazil. ${ }^{2}$ Endocrinology, Department of Internal Medicine; Clinical and Experimental Research Laboratory in Vascular Biology - BioVasc; Rua São Francisco Xavier 524, Rio de Janeiro, CEP:20550-013 - Brazil. ${ }^{3}$ Nutrition Applied Department; Nutrition Institute - Rua São Francisco Xavier, 524, Rio de Janeiro, CEP: 20550-013 - Brazil. ${ }^{4}$ Study Center for Adolescent Health - NESA Av 28 de setembro,. 87, CEP: 20551-030, Rio de Janeiro - Brazil. ${ }^{5}$ Physiological Sciences Department; Clinical and Experimental Research Laboratory in Vascular Biology - BioVasc; Rua São Francisco Xavier, 524, Rio de Janeiro CEP:20550013 - Brazil. ${ }^{6}$ Study Center for Nutrition and Oxidative Stress; Nutrition Institute; Rua São Francisco Xavier, 524, Rio de Janeiro, CEP: 20550-013 Brazil. ${ }^{7}$ Physiological Sciences and Clinical Medicine Departments; Clinical and Experimental Research Laboratory in Vascular Biology - BioVasc; Rua São Francisco Xavier, 524 Rio de Janeiro, CEP:20550-013 -Brazil.

\section{Authors' contributions}

PM - Performed microvascular analyses, statistical analyses and draft the manuscript; LGK - Participated to draft the manuscript and performed statistical analyses; CL - Participated in study design; MCK- Performed patients selection; YR - Carried out immunoassays; MG - Carried out immunoassays; JK- Performed statistical analyses and participated in study design; EB - Participated in study design and to draft the manuscript. All authors read and approved the final manuscript.

\section{Competing interests}

The authors declare that they have no competing interests.

Received: 12 February 2011 Accepted: 28 May 2011

Published: 28 May 2011

\section{References}

1. Daniels SR, Arnett DK, Eckel RH, Gidding SS, Hayman LL, Kumanyika S, et al: Overweight in children and adolescents: pathophysiology, consequences, prevention, and treatment. Circulation 2005, 111:1999-2012.

2. IBGE: Pesquisa de Orçamentos Familiares 2008-09 - Antropometria e Estado Nutricional de crianças e adolescentes do Brasil. 2010.

3. World Health Organization: Obesity: Prevention and managing: The global epidemic. Report of a WHO Consultation on Obesity 2007, Report of a WHO Consultation on Obesity..

4. Yusuf S, Hawken S, Ounpuu S, Dans T, Avezum A, Lanas F, McQueen M, Budaj A, Pais P, Varigos J, Lisheng L: Effect of potentially modifiable risk factors associated with myocardial infarction in 52 countries (the INTERHEART study): case-control study. Lancet 2004, 364:937-952.

5. Pasqui AL, Puccetti L, Di RM, Bruni F, Camarri A, Palazzuoli A, Biagi F, Servi M, Bischeri D, Auteri A, Pastorelli M: Structural and functional abnormality of systemic microvessels in cardiac syndrome X. Nutr Metab Cardiovasc Dis 2005, 15:56-64.

6. Kraemer-Aguiar LG, Maranhao PA, Cyrino FZ, Bouskela E: Waist circumference leads to prolonged microvascular reactive hyperemia response in young overweight/obese women. Microvasc Res 2010, 80(3):427-432.

7. Kraemer-Aguiar LG, Maranhao PA, Sicuro FL, Bouskela E: Microvascular dysfunction: a direct link among BMl, waist circumference and glucose homeostasis in young overweight/obese normoglycemic women? Int J Obes (Lond) 2010, 34:111-117.

8. Stapleton PA, James ME, Goodwill AG, Frisbee JC: Obesity and vascular dysfunction. Pathophysiology 2008, 15:79-89.

9. Ceriello A: Hypothesis: the "metabolic memory", the new challenge of diabetes. Diabetes Res Clin Pract 2009, 86(Suppl 1):S2-S6.

10. Roberts CK, Sindhu KK: Oxidative stress and metabolic syndrome. Life Sci 2009, 84:705-712.

11. Dandona P, Mohanty P, Ghanim H, Aljada A, Browne R, Hamouda W, Prabhala A, Afzal A, Garg R: The suppressive effect of dietary restriction 
and weight loss in the obese on the generation of reactive oxygen species by leukocytes, lipid peroxidation, and protein carbonylation. J Clin Endocrinol Metab 2001, 86:355-362.

12. Berliner JA, Heinecke JW: The role of oxidized lipoproteins in atherogenesis. Free Radic Biol Med 1996, 20:707-727.

13. Kocygit A, Koylu AA, Keles H: Effects of pistachio nuts consumption on plasma lipid profile and oxidative status in healthy volunteers. Nutr Metab Cardiovasc Dis 2006, 16(3):202-209.

14. Ros E: Nuts and novel biomarkers of cardiovascular disease. Am J Clin Nutr 2009, 89:1649S-1656S.

15. Ros E, Nunez I, Perez-Heras A, Serra M, Gilabert R, Casals E, Deulofeu R: A walnut diet improves endothelial function in hypercholesterolemic subjects: a randomized crossover trial. Circulation 2004, 109:1609-1614.

16. CDC: Center Of Disease Control and Prevention National Center For Health Statistics. CDC Growth Charts 2000; 2007.

17. Tanner J: Growth at adolescence. Oxford: Blackwell; 1962.

18. Nepa Unicamp: Tabela Brasileira de Composição de Alimentos (TACO). 2006.

19. Kraemer-Aguiar LG, Laflor CM, Bouskela E: Skin microcirculatory dysfunction is already present in normoglycemic subjects with metabolic syndrome. Metabolism 2008, 57:1740-1746.

20. Friedwald W, Levy AL, Frederickson DS: Estimation of concentrations of low density cholesterol in plasma, without use of the preparative ultracentrifuge. Clinical Chemistry 1972, 18:499-502.

21. Klyscz $T$, Junger $M$, Jung $F$, Zeintl $H$ : Cap image-a new kind of computerassisted video image analysis system for dynamic capillary microscopy. Biomed Tech (Berl) 1997, 42:168-175.

22. Ip C, Lisk DJ: Bioactivity of selenium from Brazil nut for cancer prevention and selenoenzyme maintenance. Nutr Cancer 1994, 21:203-212.

23. Thomson CD, Chisholm A, McLachlan SK, Campbell JM: Brazil nuts: an effective way to improve selenium status. Am J Clin Nutr 2008, 87:379-384.

24. Strunz CC, Oliveira TV, Vinagre JC, Lima A, Cozzolino S, Maranhao RC: Brazil nut ingestion increased plasma selenium but had minimal effects on lipids, apolipoproteins, and high-density lipoprotein function in human subjects. Nutr Res 2008, 28:151-155.

25. Ros E, Mataix J: Fatty acid composition of nuts-implications for cardiovascular health. Br J Nutr 2006, 96(Suppl 2):S29-S35.

26. Brennan AM, Sweeney LL, Liu X, Mantzoros CS: Walnut consumption increases satiation but has no effect on insulin resistance or the metabolic profile over a 4-day period. Obesity (Silver Spring) 2010, 18:1176-1182.

27. St-Onge MP: Dietary fats, teas, dairy, and nuts: potential functional foods for weight control? Am J Clin Nutr 2005, 81:7-15.

28. Rajaram S, Haddad EH, Mejia A, Sabate J: Walnuts and fatty fish influence different serum lipid fractions in normal to mildly hyperlipidemic individuals: a randomized controlled study. Am J Clin Nutr 2009, 89:1657S-1663S

29. de Jongh RT, Serne EH, ljzerman RG, Jorstad HT, Stehouwer CD: Impaired local microvascular vasodilatory effects of insulin and reduced skin microvascular vasomotion in obese women. Microvasc Res 2008 75:256-262.

30. ljzerman $R G$, de Jongh RT, Beijk MA, van Weissenbruch MM, Delemarre-van de Waal HA, Serne EH, Stehouwer A: Individuals at increased coronary heart disease risk are characterized by an impaired microvascular function in skin. Eur J Clin Invest 2003, 33:536-542.

31. Ketel IJ, Stehouwer CD, Serne EH, Poel DM, Groot L, Kager C, Hompes PG, Homburg R, Twisk JW, Smulders YM, Lambalk CB: Microvascular function has no menstrual-cycle-dependent variation in healthy ovulatory women. Microcirculation 2009, 16:714-724.

32. Fagrell $B$, Intaglietta M: The dynamics of skin microcirculation as a tool for the study of systemic diseases. Bibl Anat 1977, 231-234.

33. de Jongh RT, Serne EH, ljzerman RG, de VG, Stehouwer CD: Impaired microvascular function in obesity: implications for obesity-associated microangiopathy, hypertension, and insulin resistance. Circulation 2004, 109:2529-2535.

34. Meininger GA: Responses of sequentially branching macro- and microvessels during reactive hyperemia in skeletal muscle. Microvasc Res 1987, 34:29-45.
35. Walmsley D, Wiles PG: Reactive hyperaemia in skin of the human foot measured by laser Doppler flowmetry: effects of duration of ischaemia and local heating. Int J Microcirc Clin Exp 1990, 9:345-355.

36. Hostmarck A, Bjerkedal A, Kierulf P, Flaten H, Ulshagen K: Fish oil and plasma fibrinogen. British Medical Journal 1988, 297:180-181.

37. Lovejoy JC, Most MM, Lefevre M, Greenway FL, Rood JC: Effect of diets enriched in almonds on insulin action and serum lipids in adults with normal glucose tolerance or type 2 diabetes. Am J Clin Nutr 2002, 76:1000-1006.

38. Gabay C, Kushner I: Acute-phase proteins and other systemic responses to inflammation. N Engl J Med 1999, 340:448-454.

39. Versiek J, Cornelis R: Trace elements in plasma or serum. CRC press; 1989.

doi:10.1186/1743-7075-8-32

Cite this article as: Maranhão et al:: Brazil nuts intake improves lipid profile, oxidative stress and microvascular function in obese adolescents: a randomized controlled trial. Nutrition \& Metabolism 2011 8:32.

\section{Submit your next manuscript to BioMed Central and take full advantage of:}

- Convenient online submission

- Thorough peer review

- No space constraints or color figure charges

- Immediate publication on acceptance

- Inclusion in PubMed, CAS, Scopus and Google Scholar

- Research which is freely available for redistribution

Submit your manuscript a www.biomedcentral.com/submit
C Biomed Central 